\title{
INVESTIGATION ON THE LAST-MILE DELIVERY OF RURAL LOGISTICS UNDER CROWDSOURCING TRANSPORTATION IN CHINA
}

\author{
Qingmin Zhang1, Yisu Li ${ }^{2}$, Qianxiao $\mathrm{Cai}^{3}$ \\ 1,2,3 School of Management Science and Engineering, Nanjing University of Finance and Economics, \\ Nanjing, 3 Wenyuan Road, Qixia District, Nanjing City, Jiangsu Province, China
}

Received 17 September 2020; accepted 20 November 2020

\begin{abstract}
Recently, the last-mile delivery problem of rural e-commerce has emerged and became a bottleneck issue, due to the booming development of rural e-commerce which drives the vigorous growth of upstream agricultural products and downstream industrial products. This paper takes rural areas of China as a case study to discuss the last-mile delivery cost under crowdsourcing logistics. Owing to the absence of theoretical guidance on rural crowdsourcing logistics, the paper firstly intends to explore the theoretical framework of rural crowdsourcing logistics in terms of multi-level perspective (MLP). Then, the couriers' willingness coefficient is introduced to develop a mathematical optimization model containing time windows and vehicle capacity constraints, and the heuristic algorithm is applied to solve the model. The final results indicate that the transportation cost and Vehicle Miles Traveled (VMT) can be saved $23.85 \%$ and $37.38 \%$ respectively, compared with conventional rural logistics. Besides, analysis indicates that under certain conditions the approach of crowdsourcing delivery can be economically competitive and has valuable implication for unchoking the last-mile delivery problem of other similar rural regions.
\end{abstract}

Keywords: crowdsourcing transportation, rural logistics, last-mile delivery, cost optimization.

\section{Introduction}

Compared with city regions with full-fledged e-commerce development, rural areas have become a new blue ocean for e-commerce businesses to compete for, promoting the vigorous development of rural logistics in recent years. For example, some major provisioners in French like Carrefour, Auchan, are increasingly encouraging online sales in rural communities (Morganti et al., 2014). The Chinese government also proposed to expand the coverage of rural e-commerce and improve the three-tier logistics system of the county, township, and village (China Digital Rural Development Report, 2019). However, given rural logistics features like longer transport chains and lower consumption density, the cost of the last-mile delivery is higher, hindering logistics enterprises to the station in rural villages which becomes a restricting bottleneck for rural logistics and delivery distribution networks. Under such context, exploring how to unchoke the last-mile of rural logistics and delivery networks becomes urgent.

\footnotetext{
${ }^{2}$ Corresponding author: YisuLinufe@163.com
} 
The last-mile delivery is considered to be the most expensive (Seghezzi et al., 2020) and an inefficient part of e-commerce logistics activities (Wang et al., 2016; Akeb et al., 2018; Dupljanin et al., 2019). It is the same for the last-mile of rural logistics and delivery. The innovative approach to solve the last-mile delivery comes from two directions: transport and receipt. Existing innovative solutions for package delivery are parcel lockers within the communities (Lachapelle et al., 2018; Vakulenko et al., 2018), self-service package stations (Wang et al., 2018), self-pickup and drop-off points (Kedia et al., 2017), and neighborhood receiving points (Devari et al., 2017; Akeb et al., 2018). Besides, innovative transportation solutions mainly focus on crowdsourcing delivery with different transportation modes, including cars (Arslan et al., 2019), public buses(Gatta et al., 2019a), and bicycles (Kafle et al., 2017). The paper expects to solve the last-mile of rural logistics and delivery, so the research focuses on crowdsourcing delivery. Crowdsourcing delivery aims to outsource the last-mile delivery service to ordinary masses that will complete the package delivery from the despatch place to the destination, instead of professional full-time couriers (Mangiaracina et al., 2019). The reasons why crowdsourcing delivery can cut the cost-effectively are: (a) the reduction of fixed staff expenditure of logistics businesses, since the reduced employment rate of full-time couriers (Arslan et al., 2019) and mainly engaging part-time couriers (common people); (b) the saving of fixed transportation cost of logistics businesses, by utilizing private cars, public vehicles (Binetti et al., 2019; Gatta et al., 2019b), and taxi and others (Wang et al., 2016), instead of using special transport vehicles of logistics businesses.

However, some scholars doubted that compared with the conventional approach of full-time logistics couriers, whether crowdsourcing delivery can truly ensure lower logistics costs is worth further substantiating. The argument is that larger couriers such as FedEx and SF Express having massive parcels can reduce delivery costs by optimizing distribution routes. Comparatively, by crowdsourcing delivery, a single courier just handles a smaller amount of orders, scale economy is hardly achievable. This skepticism explained reasonably why crowdsourcing delivery has not developed and grown as some larger transport companies like SF Express.

Therefore, in the context of the rapid development of e-commerce, this paper attempted to use parcels data of courier stations in rural areas of China, and propose an optimization model of rural crowdsourcing delivery to investigate whether consolidating villagers' living paths with parcel distribution routes is conducive to saving total Vehicle Miles Traveled (VMT) and costs. Based on the existing literature (Kafle et al., 2017; Guo et al., 2019), the conceptual framework of rural logistics transformation from conventional logistics to crowdsourcing delivery is proposed. Then, with time windows and vehicle capacity limits as constraints of the proposed model, the willingness of the crowdsourcing courier is supplemented as a parameter, and the total transportation 
cost is set as objective functions. Besides, the collected information and data are input for solving the model, including longitude and latitude of villages, the number and average weight of packages for daily parcels in villages, which are obtained through field survey at specified courier stations in the rural township of China. The Part of results make comparisons of total cost and the VMT between conventional logistics and crowdsourcing logistics, to substantiate crowdsourcing's saving performance in rural areas, further verify the feasibility of crowdsourcing delivery.

The structure of the paper is constructed as follows. Part 2 is a literature review on the last-mile for rural logistics transportation and crowdsourcing delivery. Part 3 is the socio-technical theoretical framework of rural logistics in terms of multi-level perspective (MLP) and the analysis of its transformation process. Part 4 is the development of constructing and solving the optimization model of rural crowd-sourcing delivery, following the transformation principles obtained from theoretical framework analysis. Part 5 is about the design of numerical experiments to analyze the sensitivity of relevant model parameters proposed in Part 4. The final part is the conclusion and prospect of this paper.

\section{Literature Review}

The literature review focuses on two fields that are closely related to the study: (i) the study comparison of the last-mile rural logistics and delivery between developing and developed countries; (ii) major contributed research implications of crowdsourcing delivery.

Most of the existing literature on the lastmile transportation focus on cities and urban communities, and only a few literature can be the reference and theoretical basis for the research on the last-mile rural logistics and delivery. Countries in different levels of economic development confront different challenges in the development process of rural logistics, so significant differences exist between their concerning major focuses of the research (see Table 1).

\section{Table 1}

The Difference in Rural Last-mile Research Between Developing Countries and Developed Countries

\begin{tabular}{|c|c|c|c|}
\hline $\begin{array}{c}\text { Level of Economic } \\
\text { Development }\end{array}$ & Country/Region & Major Focus & Literature \\
\hline \multirow{2}{*}{$\begin{array}{c}\text { Developing } \\
\text { countries }\end{array}$} & $\begin{array}{c}\text { Republic of the } \\
\text { Congo }\end{array}$ & Medical provisions for hospitals in rural areas & Clarke et al. (2017) \\
\cline { 2 - 4 } & Africa & Absence of logistics facilities and resources & Mkansi et al. (2019) \\
\cline { 2 - 4 } & China & Quality of logistics services & Jiang et al. (2019) \\
\cline { 2 - 4 } Developed countries & Europe & $\begin{array}{c}\text { The development of logistics enterprises } \\
\text { Sher preference differences between urban } \\
\text { and rural e-commerce }\end{array}$ & Sousa et al. (2020) \\
\cline { 2 - 4 } & Sweden & $\begin{array}{c}\text { The influence of collection-and-delivery points } \\
\text { on logistics and delivery }\end{array}$ & Liu et al. (2019) \\
\hline
\end{tabular}


The logistics infrastructure in developing countries is relatively backward, and corresponding research on rural logistics focuses on how to break the situation of "starting from scratches". To solve the medication provisions interruption of rural hospitals, Clarke et al. (2017) attempted to synchronize rural hospital's medication delivery and field supervision travel routes to solve the issue that the delivery cost of medications is higher than the medications themselves. Under the constraints of synchronization, priority, and frequency of activities, they proposed the Capacitated Vehicle Routing modeling and solved the model, which made the replenishment frequency of medication provisions increased to once per month from once per four months. Jiang et al. (2019) presented that improved last-mile service quality of rural logistics and delivery is crucial to promoting the sustainable development of rural e-commerce logistics, and through the improved fuzzy analytic hierarchy process (FAHP), the interpretative structural model (ISM), and the driving force-dependency quadrant diagram, they analyzed the relationships between influence factors affecting the last-mile delivery service quality. And the results indicated that the convenience of goods changing and refunding, completeness of goods, pick-up appointment in advance, distribution cost are the utmost basic factors for sustainable last-mile delivery. Mkansi $e t$ al. (2019) believed that insufficient resources and under-developed logistics infrastructure restrained the development of e-grocery logistics in townships and villages. Moreover, they developed an APP supported township and urban e-grocery distribution models to eliminate infrastructural barriers, costs, and complexities in rural township areas. Liu
(2020) constructed the last-mile distribution routing optimization model of rural e-commerce logistics (RECL) businesses and tested five different data sets through the improved ant colony optimization to verify the effectiveness of the algorithm. Besides, the research results also reflected the impact of the number of vehicles on the maximum profit of logistics enterprises and the coverage of the RECL logistics network.

However, in developed countries, rural logistics and delivery are relatively developed at present, and they focus on the continual optimization of costs and vehicle miles traveled based on the existing rural logistics system. Sousa et al. (2020) thought that low population density and its population characteristics in rural areas made rural consumers have different online shopping preferences from urban consumers. Such differences affect the last-mile delivery cost. In the context of balancing consumer value and implementation efficiency, the strategy of providing customized delivery service should be implemented in the rural market instead of simply replicating the urban market service strategy. From the perspective of reducing VMT, Liu et al. (2019) proposed to implement the collection-and-delivery points (CDPs) to replace home delivery service; they developed a panel cross-nested logit model for individuals to decide mode options and journey routes. It was found that relocating $5 \%$ of CDPs to suburbs and rural areas could reduce the total VMT by $22.5 \%$.

According to the types of crowdsourcing courier, the literature on crowdsourcing delivery can be classified into two categories (Table 2): (i) "acquaintances", such as trustworthy friends and neighbors of 
customers' social network; (ii) “common people”, such as the general public. Both categorized literature share common points that the studies explore the issues related to transportation cost-saving and sustainable environment to a certain degree.

Table 2

Main Literature on Crowdsourcing Logistics and Transportation

\begin{tabular}{|c|c|c|}
\hline Type of Carriers & Literature and Author & Main Influence \\
\hline \multirow{2}{*}{ Acquaintances } & $\begin{array}{c}\text { Devari et al. (2017) } \\
\text { Akeb et al. (2018) }\end{array}$ & Environment, traffic congestion, economy \\
\hline \multirow{5}{*}{ Common people } & Wang et al. (2016) & Environment, social changes \\
\cline { 2 - 3 } & $\begin{array}{c}\text { Guo et al. (2019) } \\
\text { Binetti et al. (2019) } \\
\text { Dupljanin et al. (2019) }\end{array}$ & Economy, environment \\
\cline { 2 - 3 } & Giret et al. (2018) & Environment, sustainable transport \\
\cline { 2 - 3 } & Arslan et al. (2019) & Mileage savings \\
\hline
\end{tabular}

The literature on "acquaintances" delivering parcels is as follows: Devari et al. (2017) suggested consumers to ask help from friends in their social networks for realizing fast and reliable delivery. It can greatly alleviate failed delivery in virtue of "not at home" and reduce delivery cost, total air pollutant emissions, and issues like privacy protection. Akeb et al. (2018) proposed a solution based on the crowd that consists to collect and deliver parcels using individuals (neighbors). This solution can avoid the failed delivery due to customers away from home, and reduce the second delivery cost incurred extra distribution on account of the returned package.

The literature on "common people" delivering parcels is much more relatively. Some scholars paid attention to the reduction of transportation costs and VMT. Wang et al. (2016) transformed the model of large-scale traveling crowd orders to the minimum cost flow problem. They put forward various pruning technologies to significantly downsize the logistics network system and realize real-time delivery optimization, with the optimal transportation cost as the goal. Guo et al. (2019) developed the transformation framework of urban lastmile delivery from a conventional logistics network to a crowdsourcing delivery system and proposed "five principles" of the framework. They conducted the simulation to verify the applicability of the proposed theoretical framework. Their study indicated that a mixed model of crowdsourcing delivery and conventional logistics can save up to $62 \%$ of costs. Dupljanin et al. (2019) used total delivered parcels and just-in-time delivery rates to quantify the performance of crowdsourcing delivery under different transport modes for minimizing the transport cost. Arslan et al. (2019) considered adopting a crowdsourcing delivery mode consolidating the dedicated vehicles of logistics businesses and ad hoc drivers' vehicles (crowdsourcing couriers' vehicles). This model can effectively improve the costefficiency of the last-mile delivery, and save the VMT of the entire logistics network by $37 \%$, compared with the conventionally dedicated vehicle transport. 
Other scholars paid more attention to the impact of crowdsourcing delivery on the environment. Binetti et al. (2019) integrated crowdsourcing delivery and urban bikesharing system into urban freight alternative solutions to build a friendly environmental transportation mode, which serves as the supplement to local postal services. Giret et al. (2018) suggested that reducing (or even canceling) the use of dedicated vans or trucks and increasing the use of temporary couriers (traveling crowds in cities) for transportation to significantly reduce carbon dioxide emissions from freight trucks.

At present, crowdsourcing delivery has more related literature and has been widely applied in the urban last-mile delivery, but little literature is on the practice and research of crowdsourcing delivery in rural areas. However, the higher cost of rural logistics makes it difficult to complete the last-mile delivery. As mentioned in the introduction, whether crowdsourcing delivery can save costs is controversial, and the feasibility of its implementation in rural areas also remains to be studied.

\section{Theoretical Framework}

Guo et al. (2019) pointed out that the smooth transition of urban last-mile delivery from conventional logistics network to crowdsourcing delivery network belongs to the paradigm of socio-technical transformation. So it is the same for rural last-mile delivery, which requires transform the conventional logistics modes and enable villagers to enjoy the convenience of logistics and transportation technologies. From the perspective of the socio-technical paradigm, this part introduces the MLP analysis framework (Geels, 2002), and analyses the complex and dynamic transformation mechanism of rural last-mile delivery through the co-evolution relationship from three perspectives of niche, landscape, and socio-technical paradigm.

Opening the socio-technical black box of rural last-mile delivery transformation, it can be found that the rural last-mile delivery underwent three development stages as shown in Figure 1.

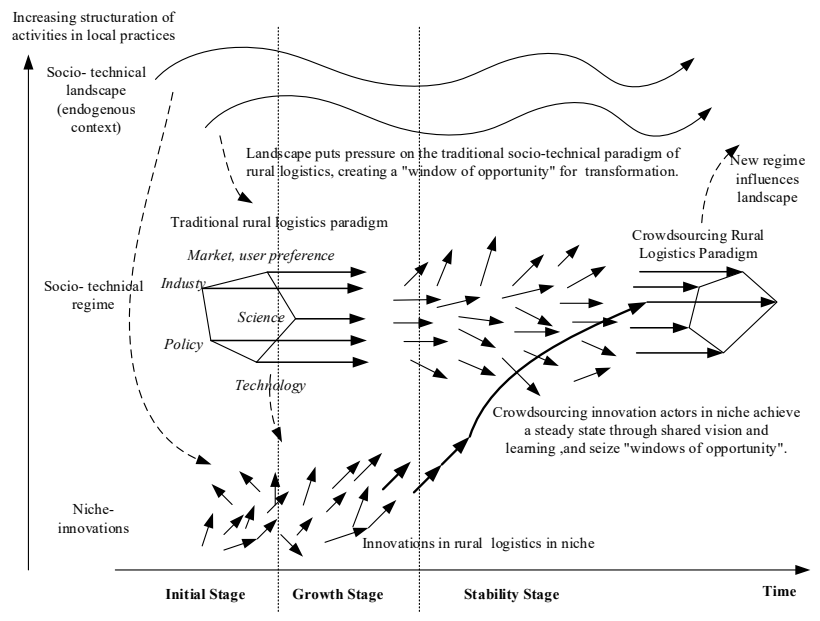

Fig. 1.

The Transformation Theoretical Framework of Rural Crowdsourcing Transportation Last-mile Delivery 
(1) Initial stage. Innovative breakthroughs of logistics and transportation modes emerged in the niche, such as crowdsourcing delivery, delivery lockers, etc. But such niches are still fragile and the technical cost is higher. They hardly pose a threat to the conventional rural logistics paradigm outside the niches. Therefore, the principles of "small-scale pilot" and "low additional investment" should be followed in the initial stage of rural crowdsourcing delivery.

As for the principle of rural "small-scale pilot", given that crowdsourcing delivery use common people as couriers, consumer privacy and package security still need to be improved, and acceptability of customers to crowdsourcing delivery service is low, so it is applicable to carry out smaller-scale pilot during the initial stage regard to this study. The experimental scope in the case study of this paper is limited to certain rural areas in China, not cover the entire market. As for the principle of "low additional investment", it means that the transformation of rural logistics will utilize the existing resources utmostly, rather than rebuild resources, which can increase the success rate of crowdsourcing operation. For example, in terms of information technology, it can cooperate with existing takeaway platforms such as Meituan to deliver and track packages.

(2) Growth stage. Within the protected space in the niche, the crowdsourcing socio-technical paradigm gradually forms a stable structure undergoing constant trials and practice. At the same time, the rapid development of e-commerce continues to impose pressure on conventional rural logistics, resulting in the cracks of its internal structure and providing a "window of opportunity" for the realization of crowdsourcing innovation.
In this stage, rural crowdsourcing delivery needs to follow the principle of "low network complexity", avoid increasing the complexity of the existing delivery network, and further reduce the complexity of rural crowdsourcing delivery under "window of opportunity". Ideally, allowing transportation between couriers would expand potential crowdsourcing crowds, but this is based on the advanced level of the Internet of things (IoT) technology and will greatly increase the complexity of the delivery network. Therefore, crowdsourcing delivery networks should be simple as much as possible, for example, a single crowdsourcing courier should finish transportation and multiple delivery operations. To meet the principle of low network complexity, the proposed model of this paper assumes that only one crowdsourcing courier is responsible for the parcels, and one courier can deliver multiple parcels.

(3) Stability stage. In this stage, the dominant conventional rural logistics and delivery continue to loosen. For example, the government encourages the intensive development mode of reducing cost and increasing efficiency (E-commerce in China 2019). Enterprise strategy and investment direction gradually extended to rural areas, so that the original industrial alliance began to collapse and reconstruct. Crowdsourcing niches have achieved fullfledged breakthroughs and can compete with conventional rural logistics. Finally, crowdsourcing technology and logistics modes win the recognition, and the matched crowdsourcing socio-technical paradigm becomes the dominant paradigm for rural logistics and delivery, and replace the conventional rural logistics socio-technical paradigm, thus crowdsourcing delivery transformation is completed. 
In this stage, the principle of "pick-up and drop-off en route" should be followed. On the living routes to and from villages (commuting, shopping, etc.), to meet consumer demand and realize corporate revenue targets, the extra cost of picking up and delivering parcels should be slightly increased. In this paper, the rural crowdsourcing distribution route is established on the life route of villagers to and from villages and townships, to realize parcel delivery, which conforms to the principle of "pick-up and drop-off en route".

\section{Methodology}

\subsection{Problem Overview}

In this paper, the rural last-mile delivery refers to the parcel transportation between villages and townships. No matter from the view of the consignors or consignees, the transportation costs of conventional rural logistics become a great obstacle for e-commerce operators stationing in villages and squeeze out the profit of e-commerce. From the perspective of the consignees, the higher logistics cost of rural last-mile delivery is owing to the long traveling distance and the spatial-temporal dispersion of customers' demands, which result in the transportation cost is even higher than the revenue. Besides, it is also the reason why logistics enterprises are difficult to station in villages and townships. From the perspective of the consignors, the high cost of rural logistics in China is due that the most of parcels are only delivered to courier stations of townships, the villagers had to walk a long distance and pick up their parcels from courier stations or pay others to fetch on behalf of them (the commission is sometimes as high as $1.3 €$ per parcel).

Additionally, the rural crowdsourcing delivery optimization model is constructed in this part of the paper (see Figure 2). The details of the implementation process are as follows: villagers, who are consignors and have delivery service demands, announce the related information concerning on the parcels. Then, the crowdsourcing courier compares the mileage they will travel and the cost between their original path (dotted arrow) and crowdsourcing paths (solid arrow), to decide whether to accept the orders. After accepting delivery orders, the crowdsourcing courier goes to the courier station (white rectangle) to pick up the parcels, then send it to the consignors, and finally back to the destination (blue rectangle). On the contrary, if the crowdsourcing courier does not accept the delivery orders, they return to their destination along the black dotted line. 


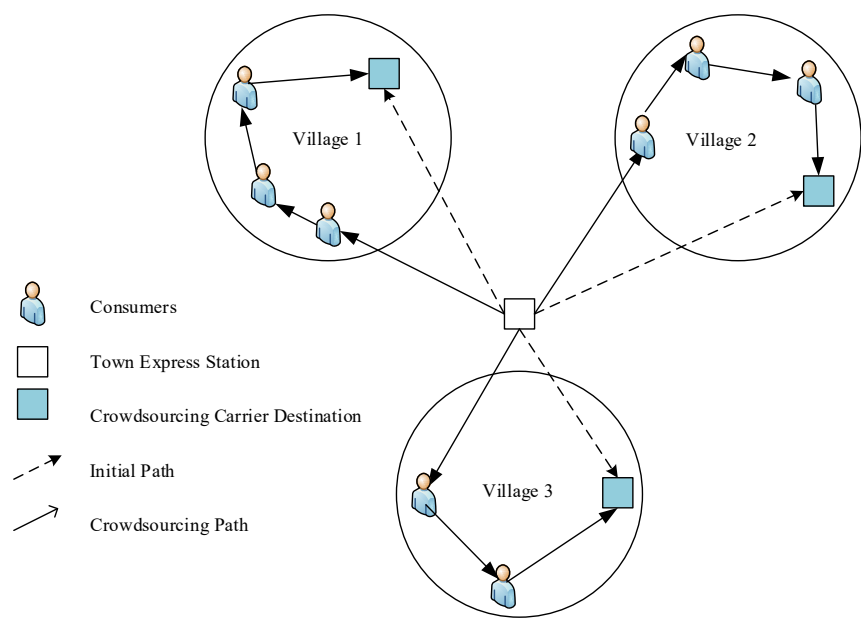

Fig. 2.

Rural Crowdsourcing Delivery Optimization Model

\subsection{Mathematical Formulation}

This model addressed in the study is a multiobjective programming (MOP) problem with constraints of time windows and vehicle capacity. Heuristic algorithms are used to solve the model, to minimize the total cost of the logistics network and maximize consumer satisfaction (meeting time window requirements). The assumptions of the model are as follows:

- Express consignments are massive and various. For convenience, the consigned goods are regarded as the same quality volume parcel;

- The location of each consumer presence is known and represented by latitude and longitude of the location;

- Crowdsourcing order information has been released on the crowdsourcing platform in advance;

- Each consumer is served only once;
- Each consumer has their delivery service time requirement, and the time window is known, including preparation time;

- The maximum loaded capacity of each courier is not distinguished.

When traveling on the roads, vehicles will encounter traffic jams or accidents with a certain probability, and characteristics of goods make consumers have different requirements for delivery time. Some goods like fresh products, medicines, food (Sousa et al., 2020), have more stringent requirements on the delivery time. Therefore, time windows are set to constrain the timeliness and efficiency of the crowdsourcing courier. The crowdsourcing courier should complete the delivery service within the time window expected by the customers. If the crowdsourcing courier arrives earlier to the consumer location, then had to wait. If the 
crowdsourcing courier arrives later after the consumer expected time window, it will result in poor customer satisfaction for the delivery service, which is not conducive to building consumer trust in crowdsourcing transportation. Therefore, in the model design to control the crowdsourcing courier timeout behaviour, the courier who violates the time window constraint will be subject to a certain penalty(deduct money).

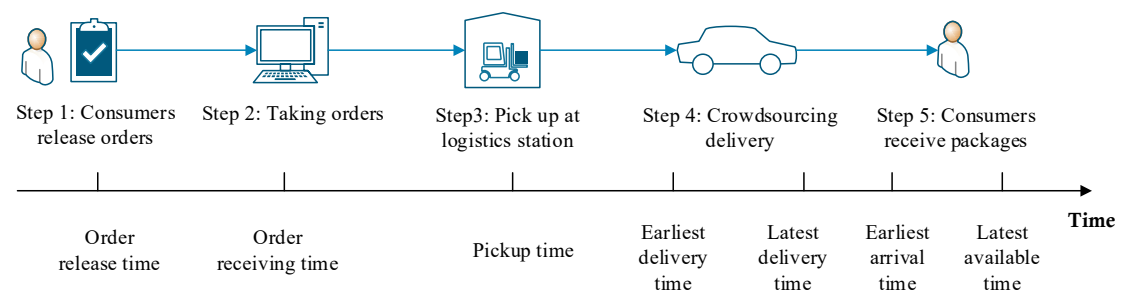

Fig. 3.

Rural Crowdsourcing Delivery Steps and Timeline

The operation timeline of rural probability during couriers traveling. crowdsourcing delivery is shown in Therefore, the earliest and the latest Figure 3. First, consumers release order departure time of the courier leaving the information. Then, after taking the order, courier station is set. The latest arrival time the crowdsourcing courier will go to the to the consumer location must be no later courier station to pick up the parcels. Some than the expected earliest time to serve the emergencies may happen with certain consumer present in the time window.

The complete formulation expression of the problem is given below. Let:

Sets:

0 Courier station;

$\mathrm{N} \quad$ Set of consumers $n \in\{1,2, \ldots, N\}$;

$\mathrm{K}$ Set of Crowdsourcing courier vehicles $k \in\{1,2, \ldots, K\}$;

A Set of Crowdsourcing courier at initial points $a \in\{1,2, \ldots, A\}$;

B Set of Crowdsourcing courier at destinations, $b \in\{1,2, \ldots, B\}$;

Note: The distance difference between courier stations in the town is small, so 0 is used to represent all courier stations.

Parameters:

$i \quad$ Longitude;

j Latitude; 
$m_{1}$ The average traveling cost of the vehicle per mile, proportional to mileage traveled;

$m_{2}$ Crowdsourcing courier service cost per unit of time;

$C_{1}$ Crowdsourcing courier travel costs;

$C_{2}$ Crowdsourcing courier service costs, including travel costs and loading and unloading costs;

$\varepsilon \quad$ Average traveling and loading times;

$V$ Average travel speed of crowdsourcing courier;

$d_{i j}$ Distance from the point $i$ to point $j$;

$E_{n}$ The earliest time allowed arriving at the customer location;

$L_{n}$ The latest time allowed arriving at the customer location;

$f$ Delay penalty;

$A t_{n}^{k}$ The actual arrival time of crowdsourcing courier $\mathrm{k}$ to reach consumer $\mathrm{n}$;

$q_{n}$ The demands of the nth consumer;

$Q_{k}$ Crowdsourcing courier vehicle capacity;

$\beta$ Willingness coefficient of crowdsourcing courier to serve consumers;

Decision Variables:

$$
\begin{aligned}
x_{i j}^{k} & =\left\{\begin{array}{l}
1, \text { Carrier } k \text { travels from } i \text { to } j \\
0, \text { Otherwise }
\end{array}\right. \\
y_{n}^{k} & =\left\{\begin{array}{l}
1, \text { When con sumer } n \text { is served by carrier } k \\
0, \text { Otherwise }
\end{array}\right. \\
w^{k} & =\left\{\begin{array}{l}
1, \text { Carrier } k \text { complete delivery within the time window } \\
0, \text { Otherwise }
\end{array}\right.
\end{aligned}
$$


Objective Function:

Minimize $Z_{1}=\sum_{k=1}^{K} \sum_{i=0}^{N+A+B} \sum_{j=0}^{N+A+\mathrm{B}}\left[m_{1} \cdot d_{i j} \cdot x_{i j}^{k}+\varepsilon \cdot m_{2} \cdot\left(x_{i j}^{k}-1\right)\right]$

$\operatorname{Maximize}_{2}=\frac{\left(\sum_{i=1}^{N} y_{n}^{k}\right)}{n}$

Subject to:

$\sum_{n=1}^{N} y_{n}^{k} q_{n} \leq Q_{k}, k=1,2, \ldots K$

$A t_{n}^{k}=\frac{\sum_{a=1}^{K} \sum_{b=1}^{K} \sum_{n=1}^{N}\left(d_{a 0}+d_{0 n}+d_{i j}\right) \cdot x_{i j}^{k}}{v}+\varepsilon \cdot \sum_{k=1}^{K}(n+1) \cdot y_{n}^{k}$

$E_{n} \leq A t_{n}^{k} \leq L_{n}, n=0,1, \ldots, N$

$\sum_{k=1}^{K} y_{n}^{k}=1, n=1,2, \ldots, N$

$\sum_{k=1}^{K} x_{n b}^{k}=1, n=1,2, \ldots, N$

$x_{i j}^{k} \in\{0,1\}, \forall k \in K$

$y_{n}^{k} \in\{0,1\}, \forall k \in K, n=1,2, \ldots, N$

$w^{k} \in\{0,1\}, \forall k \in K$

$d_{a 0}+d_{0 n}+d_{n b} \leq \beta d_{a b},(\beta>1)$

The objective function of the model (1) is to minimize the total transportation cost $C$, which is divided into crowdsourcing courier traveling cost $C_{1}$ and crowdsourcing courier service cost $C_{2}$. Assuming that the crowdsourcing courier takes a fuel-based 
vehicle as transportation means, the traveling cost is related to the traveling time of the crowdsourcing courier, and the fuel cost per mile $m_{1}$ is used to represent the traveling cost $C_{1}=m_{1} \cdot \sum_{k=1}^{K} \sum_{i=0}^{N} \sum_{j=0}^{N} d_{i j} \cdot x_{i j}^{k}$.

The consumer service cost $C_{2}$ consists of two components: walking costs and loading and unloading costs, (a) Walking costs: rural roads are narrow, and residencies of some consumers are not along the main roads. Crowdsourcing courier must drive their vehicles a certain distance to complete package delivery; (b) Loading and unloading costs: it incurs when the crowdsourcing courier to pick up the parcels at the courier station, and drop off the parcels at the delivery destination. Since the two kinds of costs are usually incurred at the same time, in the model, the service cost per unit time is expressed as $m_{2}$, and the consumer service cost is expressed as

$$
C_{2}=m_{2} \cdot \varepsilon \cdot \sum_{k=1}^{K} \sum_{i=0}^{N} \sum_{j=0}^{N}\left(x_{i j}^{k}-1\right)
$$

The objective function (2) is a satisfaction function, indicating that the vehicle meets the consumer's expected time window requirements for delivery service as much as possible. Under constraint conditions, constraint (3) indicates that the demands of each consumer cannot exceed the maximum load of the crowdsourcing courier's vehicle. Constraint (4) indicates that the arrival time of parcels to reach the consumer, which is the time duration from the crowdsourcing courier picks up the parcels at the courier station till the parcels are delivered to the consumer. Constraint (5) indicates that the delivery time of parcels should be within the required time of delivery service. Constraint
(6) indicates that the crowdsourcing courier serves customer, each consumer is served by a crowdsourcing carrier, and there is no unattended order. Constraint (7) indicates that all crowdsourcing couriers will return to their destination eventually. Constraints (8-10) are standard integrality constraints and the variables in that are binary. Finally, constraint (11) is used to constrain the delivery range of the crowdsourcing carriers. In general, the carrier is unwilling to accept the order requiring longer travel distance even if the customer pays a higher commission for it. Here, $\beta$ represents the willingness of the crowdsourcing courier to conduct the crowdsourcing order, if the value is greater than 1 , it means that the crowdsourcing courier who engages in crowdsourcing delivery needs to travel more miles than those without crowdsourcing transportation.

\section{Case Analysis}

\subsection{Computing Test}

To explore the feasibility of crowdsourcing delivery in rural areas and eliminate logistics and delivery barriers between townships and villages, this part conducts a case study. The data is acquired from GIS and Town A's courier stations in the north of Jiangsu Province. Located at N32 $43^{\circ}$ ' $29^{\prime}$ " $\sim 33^{\circ} 11^{\prime}$ ' 04 ” and W $118^{\circ} 11^{\prime} 43^{\prime \prime} \sim 118^{\circ} 54^{\prime}$ ' $26^{\prime}$ ”, the county has a population of 804,700 and the surface area of 2497.3 square kilometers (360 Encyclopedias, 2016). In 2019, the data of China E-commerce report (E-commerce in China, 2019) shows that the number of rural Internet users in China has reached 255 million, accounting for $18.2 \%$ of the total population of China (National Bureau of Statistics, 2010). Therefore, it can be calculated that the number of rural 
Internet users in Town A is 4,040 assumedly. According to the survey, the average distance between Town A and courier stations is about $9.1 \mathrm{~km}$. In terms of Chinese traffic regulations, $40 \mathrm{~km} / \mathrm{h}$ is set as the driving speed of the crowdsourcing courier's vehicle in the model, so the average travel time is about $14 \mathrm{mins}$. The average service time to deliver the parcels to consumers is set as $13 \mathrm{~min}$, and the delay penalty is set as $20 \mathrm{RMB}$ per order by reference to the Meituan standard (a well-known takeaway platform in China). According to the pricing rules of Didi (similar to Uber), the unit traveling cost is $0.0733 € / \mathrm{km}$ and service cost is $0.0038 € /$ min. The success of crowdsourcing delivery depends on whether the service platform has enough crowds. The survey found that more than $60 \%$ of crowds are willing to engage in crowdsourcing delivery activities (Punel and Stathopoulos, 2017), it can work out that Town A has at least 2,424 potential crowdsourcing couriers and 0.16 parcels per person per day (total 326 parcels) averagely, which is relatively small compared with 2,424 potential couriers. Therefore the crowds can support the operation of crowdsourcing delivery in rural markets. According to the above analysis, all parameters of the experiment are obtained (see Table 3).

\section{Table 3}

Experimental Parameters

\begin{tabular}{|c|c|c|}
\hline Parameter & Unit & Value \\
\hline The proportion of rural internet users & - & $18.2 \%$ \\
\hline The number of internet users & Person & 4040 \\
\hline Speed & $\mathrm{km} / \mathrm{h}$ & 40 \\
\hline Unit traveling cost & $€ / \mathrm{km}$ & 0.0733 \\
\hline Service time & $\mathrm{min}$ & 13 \\
\hline Unit service cost & $€ / \mathrm{min}$ & 0.0038 \\
\hline
\end{tabular}

\subsection{Results}

This section takes the total transportation cost and miles saving ratio as indicators. The total transportation cost directly affects the feasibility of rural crowdsourcing delivery, and indirectly affects the willingness of enterprises and villagers to engage in crowdsourcing activities. Most cost of crowdsourcing delivery comes from the increase of VMT, so the total transportation cost and miles saving ratio are selected as indicators, and the self-pickup package cost and miles of conventional rural logistics are used as the baseline experiment.

\subsubsection{Conventional Logistics and Crowdsourcing}

All comparative analyses of examples are conducted under the environment of the $2.5 \mathrm{GHz}$ Intel Core i5 processor and $8 \mathrm{~GB} 1867 \mathrm{MHz}$ DDR3 of installed RAM MATLAB2016b. The parameters in Table 3 are substituted into the model to compute the solution, and the results are shown in Table 4. 
Table 4

Computing Results for Conventional Rural Logistics and Crowdsourcing Rural Delivery (per day)

\begin{tabular}{|c|c|c|}
\hline Type of Transport & Total VMT $(\mathbf{k m})$ & Total Cost $(\boldsymbol{\epsilon})$ \\
\hline Conventional rural logistics & 3060.57 & 128.5151 \\
\hline Crowdsourcing Rural delivery & 1916.54 & 97.8613 \\
\hline
\end{tabular}

From Table 4, it can be seen that rural crowdsourcing delivery is superior to conventional rural logistics in terms of total VMT, with the former saving $37.38 \%$ of VMT compared with the latter. From the perspective of the total cost, rural crowdsourcing delivery can save up to $23.85 \%$ compared with conventional rural logistics. The above results show that rural crowdsourcing delivery is much superior to conventional rural logistics in terms of VMT and cost, and is applicable and stable.

\subsubsection{Parameter Analysis}

(1) The influence of the same delivery willingness of couriers on total VMT and total cost if couriers use a different type of vehicles.
In practice, the vehicle types are varied, so the vehicle weight limit and speed will change accordingly, affecting logistics cost. Vehicle weight limit restricts the loaded weight of crowdsourcing carriers, that is, the maximum weight of a single parcel satisfying the demand of consumers. In urban logistics, the greater the delivery willingness is, that is, the farther the traveling distance is, the greater the income will be. Further analysis remains to see whether the same conclusion can be drawn in rural logistics. Therefore, the sensitivity analysis experiment is designed and conducted to observe the influence of varied vehicles with the same delivery willingness on total VMT and total cost, including electric bicycles, motorcycles, and cars with a corresponding weight limit of $20 \mathrm{~kg}, 40 \mathrm{~kg}, 60 \mathrm{~kg}$ and corresponding driving speed at $30 \mathrm{~km} / \mathrm{h}, 50 \mathrm{~km} / \mathrm{h}, 50 \mathrm{~km} / \mathrm{h}$.

Table 5

Total Cost and Total VMT of Different Vehicle Types

\begin{tabular}{|c|c|c|c|}
\hline Vehicle Types & Cars (Vehicle 1) & Motorcycles (Vehicle 2) & Electric Bicycles (Vehicle 3) \\
\hline VMT $(\mathrm{km})$ & 1916.54 & 1915.98 & 2399.45 \\
\hline Total Cost $(€)$ & 87.4556 & 87.4350 & 16.3492 \\
\hline
\end{tabular}

From the results in Table 5, it can be seen that cars and motorcycles are relatively economical in terms of VMT, and the gap between them is small because the vehicles' driving speeds are the same. Compared with conventional rural logistics, $37.38 \%$ and $37.4 \%$ of the VMT are achieved respectively. However, electric bicycles only achieved $21.6 \%$ of the VMT, inferior to cars and motorcycles. It indicates that if conditions are allowable, it is better to choose fuel-based vehicles for crowdsourcing delivery. In terms of the total cost, although the results are obvious, there is still necessary to validate. The cost of electric bicycles is significantly lower than that of cars and motorcycles, saving up to $87.28 \%, 31.95 \%$, and $31.97 \%$ respectively. The miles saving of electric bicycles are low relatively, but its costefficiency performance is very significant. 
(2) Impact of the different delivery willingness of couriers on total VMT and total cost if they use the same type of vehicles.

Suppose the delivery willingness changes within the interval $[1.5,2.5]$, the step length of willingness is incremental with 0.1 , and the types of vehicles are still the same as the above. By the observance of computed total cost and total VMT, the following phenomenon is noticed.

When the delivery willingness $[1.5,1.9]$, the number of orders undertaken by the couriers is constant, no matter what types of vehicles are, delays exist. When the

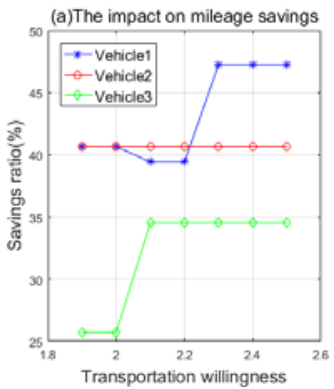

delivery willingness is low, the transport range of couriers is narrow down. Because the number of undertaken orders and the number of couriers remains the same, it means an increase in the number of orders to be transported by one carrier, equating to an increase in demand density. When the demand density increases, the time of services also increases because each order has a fixed service time and travel time so that the carriers have to take up other times leading to exceeding the time window. Thus, with a limited number of couriers and high demand density, transportation time windows should be rationalized according to the urgency of the consumer demand to increase consumer satisfaction.

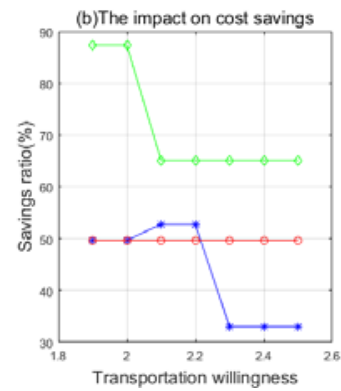

Fig. 4.

Influence of Vehicle Type and Transport Willingness on Savings of Total Cost and Total Mileage

Excluded outliers (the timeout behavior), the three types of vehicle VMT and cost in transportation will near 2.3 to reach a steady-state (Figure 4). It can be seen from Figure 4 (a), with the increase of delivery willingness, the VMT savings of cars and electric bicycles increased significantly. However, the VMT savings of motorcycles did not change significantly, and the VMT saving of cars is better than that of the other two types of vehicles. It can be seen from Figure 4 (b) that, with the increase in delivery willingness, the cost savings of cars and electric bicycles decrease sharply, and the cost-saving of motorcycles is the opposite. The cost savings for electric bicycles are much higher than those of the other two types of vehicles. Thus, it can be concluded that the courier delivery willingness is less sensitive to the impact of motorcycle cost and VMT, and electric bicycle transport has an obvious advantage in cost-saving.

Therefore, cars have the best performance on VMT saving but higher consumed cost. Motorcycles have an intermediate performance 
on cost and VMT savings. Since both vehicles are fuel-based and emit greenhouse gas during driving, the principle of "collaborative functionality" cannot be well implemented. Electric bicycles' saving of VMT is relatively low, but its overall performance is still better than conventional rural logistics. Therefore, it is suggested that electric bicycles should be selected as the main transport means when the courier engage in rural crowdsourcing delivery. To serve consumers of crowdsourcing delivery requiring distant VMT, it is recommended to utilize cars to avoid detours for saving time and travel distance.

\section{Conclusions}

The present paper studies the application of crowdsourcing in rural last-mile delivery, and the research provides theoretical and practical contributions. In terms of academic theories, the theoretical framework of rural crowdsourcing delivery is deepened through the theory of socio-technical transformation from MLP. Three different stages of rural crowdsourcing delivery development, including initial stage, growth stage, and stability stage are analyzed respectively, and it is concluded that the transformation needs to follow four principles of "smallscale pilot", "low additional investment", "low network complexity", and "pick-up and drop-off en route", from rural conventional logistics to the rural crowdsourcing delivery. In practice, this paper carried out a case study of Chinese rural crowdsourcing delivery. Professional logistics transportation service is unavailable in rural townships, so the conventional rural logistics confront difficulties of smooth circulation upstream and downstream. Considering such problem, this paper establishes a low-cost rural crowdsourcing delivery optimization model and utilizes "common people" who travel back and forth villages in their daily life to pick up and deliver the parcels, thus realize the rural last-mile delivery. Such research has not been explored before. To solve the rural last-mile delivery problem of crowdsourcing delivery in rural areas with constraints of the time window and vehicle capacity, a mathematical model is constructed and a heuristic algorithm is applied to solve the model. Numerical experimentation and sensitivity analysis verify the effectiveness of the model in real situations. The advantages of this model are that it effectively integrates the life path of ordinary people and delivery routing, while capturing the complexity and issues involved in the processes of crowdsourcing delivery, such as vehicle capacity constraints, limited carriers delivery willingness range, and time window requirements. Besides, rural crowdsourcing delivery has significant savings in cost and VMT compared with conventional rural logistics, and this advantage has significant implications for other rural areas under a similar situation.

Last, crowdsourcing delivery in rural areas can effectively save VMT, which means it can also effectively reduce pollutant emissions of vehicles. At present, a friendly environmental society is advocated over the world. In this paper, the impact of mileage saving on a sustainable environment has not been discussed. In the future, relevant parameters such as carbon emissions can be added based on the proposed model in this paper to explore the total energy consumption and carbon emissions under two different transportation modes, conventional logistics, and rural crowdsourcing delivery. Also, with the continuous development of the economy in the future, UAV (unmanned aerial vehicle) and other high-tech technologies will gradually enter people's lives. UAV transport, 
powered by electricity at a lower cost, is also a promising and innovative way to solve the rural last-mile delivery problem.

\section{Acknowledgments}

The paper was done within the research projects (20BGL182): Mechanism, path, and effect of sustainable development of rural e-commerce clusters in China under digital rural strategy, which was funded by The National Social Science Fund of China (NSSFC). Also, the authors are grateful for the support and help of the Courier station manager.

\section{References}

Akeb, H.; Moncef, B.; Durand, B. 2018. Building a collaborative solution in dense urban city settings to enhance parcel delivery: An effective crowd model in Paris, Transportation Research Part E-Logistics and Transportation Review 119: 223-233.

Arslan, A. M.; Agatz, N.; Kroon, L.; Zuidwijk, R. 2019. Crowdsourced Delivery-A Dynamic Pickup and Delivery Problem with Ad Hoc Drivers, Transportation Science 53(1): 222-235.

Binetti, M.; Caggiani, L.; Camporeale, R.; Ottomanelli, M. 2019. A Sustainable Crowdsourced Delivery System to Foster Free-Floating Bike-Sharing, Sustainability 11(10): 2772-2795.

China Digital Rural Development Report. 2019. Agricultural and Rural Informatization Expert Advisory Committee. Available from Internet: <http://www.moa. gov.cn/xw/bmdt/201911/P020191119505821675490.pdf >.

Clarke, J.; Gascon, V.; Ferland, J. A. 2017. A capacitated vehicle routing problem with synchronized pick-ups and drop-offs: the case of medication delivery and supervision in the DR Congo, IEEE Transactions on Engineering Management 64(3): 327-336.
Devari, A.; Nikolaev, A. G.; He, Q. 2017. Crowdsourcing the last-mile delivery of online orders by exploiting the social networks of retail store customers, Transportation Research Part E: Logistics and Transportation Review 105: 105-122.

Dupljanin, D.; Mirkovic, M.; Dumnic, S.; Culibrk, D.; Milisavljevic, S.; Sarac, D. 2019. Urban crowdsourced last-mile delivery: mode of transport effects on fleet Performance, International Journal of Simulation Modelling 18(3): 441-452.

E-commerce in China. 2019. Department of E-commerce and Informatization, Ministry of Commerce. Available from Internet: < http://www.gov.cn/ xinwen/2020-07/02/5523479/files/0a2c57d8ba6d4e 26b83d96cdd764d6f0.pdf $>$.

Gatta, V.; Marcucci, E.; Nigro, M.; Patella, S.M.; Serafini, S. 2019a. Public transport-based crowdshipping for sustainable city logistics: assessing economic and environmental impacts, Sustainability 11(1): 145-158.

Gatta, V.; Marcucci, E.; Nigro, M.; Serafini, S. 2019b. Sustainable urban freight transport adopting public transport-based crowdshipping for B2C deliveries, European Transport Research Review 11(1): 1-14.

Geels, F.W. 2002. Technological transitions as evolutionary reconfiguration processes: a multi-level perspective and a case-study, Research Policy 31(8): 1257-1274.

Giret, A.; Carrascosa, C.; Julian, V.; Rebollo, M.; Botti, V. 2018. A crowdsourcing approach for sustainable lastmile delivery, Sustainability 10(12): 4563-4579.

Guo, X.; Jaramillo, Y.J.L.; Bloemhof-Ruwaard, J.; Claassen, G.D.H. 2019. On integrating crowdsourced delivery in last-mile logistics: A simulation study to quantify its feasibility, Journal of Cleaner Production 241: 118365-118377. 
Jiang, X.; Wang, H.; Guo, X.; Gong, X. 2019. Using the FAHP, ISM, and MICMAC approaches to study the sustainability influencing factors of the last-mile delivery of rural e-commerce logistics, Sustainability 11(14): 3937.

Kafle, N.; Zou, B.; Lin, J. 2017. Design and modeling of a crowdsource-enabled system for urban parcel relay and delivery, Transportation Research Part B-Methodological 99: 62-82.

Kedia, A.; Kusumastuti, D.; Nicholson, A. 2017. Acceptability of collection and delivery points from consumers' perspective: A qualitative case study of Christchurch city, Case Studies on Transport Policy 5(4): 587-595.

Lachapelle, U.; Burke, M.; Brotherton, A.; Leung, A. 2018. Parcel locker systems in a car dominant city: Location, characterisation and potential impacts on city planning and consumer travel access, Journal of Transport Geography 71: 1-14.

Liu, C.; Wang, Q.; Susilo, Y.O. 2019. Assessing the impacts of collection-delivery points to individual's activity-travel patterns: A greener last-mile alternative? Transportation Research Part E-Logistics and Transportation Review 121: 84-99.

Liu, W. 2020. Route Optimization for Last-Mile Distribution of Rural E-Commerce Logistics Based on Ant Colony Optimization, IEEE Access 8: 12179-12187.

Mangiaracina, R.; Perego, A.; Seghezzi, A.; Tumino, A. 2019. Innovative solutions to increase last-mile delivery efficiency in B2C e-commerce: a literature review, International Journal of Physical Distribution \& Logistics Management 49(9): 901-920.

Mkansi, M.; de Leeuw, S.; Amosun, O. 2019. Mobile application supported urban-township e-grocery distribution, International Journal of Physical Distribution \& Logistics Management 50(1): 26-53.

Morganti, E.; Dablanc, L.; Fortin, F. 2014. Final deliveries for online shopping: The deployment of pickup point networks in urban and suburban areas, Research in Transportation Business \& Management 11: 23-31.

National Bureau of Statistics. 2010. The 6th census. Available from Internet: <http://www.stats.gov.cn/ tjsj/pcsj/rkpc/6rp/indexch.htm $>$.

Punel, A.; Stathopoulos, A. 2017. Modeling the acceptability of crowdsourced goods deliveries: Role of context and experience effects, Transportation Research Part E: Logistics and Transportation Review 105: 18-38.

Seghezzi, A.; Mangiaracina, R.; Tumino, A.; Perego, A. 2020. 'Pony express' crowdsourcing logistics for last-mile delivery in B2C e-commerce: an economic analysis, International Journal of Logistics Research and Applications 1-17. doi: https://doi.org/10.1080/13675 567.2020.1766428.

Sousa, R.; Horta, C.; Ribeiro, R.; Rabinovich, E. 2020. How to serve online consumers in rural markets: Evidence-based recommendations, Business Horizons 63(3): 351-362.

Vakulenko, Y.; Hellstrom, D.; Hjort, K. 2018. What's in the parcel locker? Exploring customer value in e-commerce last-mile delivery, Journal of Business Research 88: 421-427.

Wang, X.; Yuen, K.F.; Wong, Y.D.; Teo, C.C. 2018. An innovation diffusion perspective of e-consumers' initial adoption of self-collection service via automated parcel station, International Journal of Logistics Management 29(1): 237-260.

Wang, Y.; Zhang, D.; Liu, Q.; Shen, F.; Lee, L.H. 2016. Towards enhancing the last-mile delivery: An effective crowd-ordering model with scalable solutions, Transportation Research PartE-Logistics and Transportation Review 93: 279-293.

360 Encyclopedias. 2016. The County of Xuyi in Huai 'an, Jiangsu Province. Available from Internet: <https:// baike.so.com/doc/4451077-4659538.html>. 